\title{
Structured information in small-world neural networks
}

\author{
David Dominguez, * Mario González, Eduardo Serrano, and Francisco B. Rodríguez \\ EPS, Universidad Autónoma de Madrid, 28049 Madrid, Spain
}

(Received 29 July 2005; revised manuscript received 18 August 2008; published 10 February 2009)

\begin{abstract}
The retrieval abilities of spatially uniform attractor networks can be measured by the global overlap between patterns and neural states. However, we found that nonuniform networks, for instance, small-world networks, can retrieve fragments of patterns (blocks) without performing global retrieval. We propose a way to measure the local retrieval using a parameter that is related to the fluctuation of the block overlaps. Simulation of neural dynamics shows a competition between local and global retrieval. The phase diagram shows a transition from local retrieval to global retrieval when the storage ratio increases and the topology becomes more random. A theoretical approach confirms the simulation results and predicts that the stability of blocks can be improved by dilution.
\end{abstract}

DOI: 10.1103/PhysRevE.79.021909

PACS number(s): 87.18.Sn, 89.75.Fb, 05.10.-a, 64.60.Cn

\section{INTRODUCTION}

Attractor neural networks (ANNs) often deal with global overlapping between patterns and neural states, employing uniform connectivity to perform retrieval tasks. They are useful when the information involved is spatially distributed because pattern learning is resistant to damage of parts of the network [1]. When these networks start from only local stimuli, however, no global information can be achieved.

More structured ANN architectures have recently been studied, especially small-world topologies [2-4]. Such graphs are modeled by two parameters, the connectivity and the randomness of the links, and they can capture most features of a wide range of networks $[5,6]$. The retrieval ability of such networks are commonly measured by the overlap between neuron states and memorized patterns, and the load parameter expressed as the ratio between stored patterns and links per node [7]. Indeed, above a critical value of the load parameter no retrieval is possible and the overlap goes to zero. In addition, the mutual information between the stored patterns and the neural states has been proposed to compare the performance of different topologies in terms of memory retrieval $[4,8,9]$. Although the critical load increases monotonically with dilution and randomness, the information is a nonmonotonic function of the load and reaches a maximum that corresponds to a nontrivial optimal topology [4].

Most studies into associative memory networks with spatial structure [3,10-13] focus on the global retrieval of a pattern, without considering the possibility of spatially localized states. In general, these papers deal with the relation between the storage capacity and the degree of randomness in the network. The only source of information in a longrange connected ANN (either fully connected or random), is the standard uniformly distributed overlap along the network. However, for spatially structured topologies one might measure local overlaps inside blocks of contiguous neurons.

Although the information about a pattern is invariant under the reverse transformation of the global overlap, it vanishes if only half of the neuron states are flipped. If we sup-

\footnotetext{
*david.dominguez@uam.es
}

pose a case where sequential blocks of pixels of a binary image are flipped, the overall pattern is probably still recognizable. As an example, Fig. 1 shows a natural image in black and white pixels in the left panel, while the central panel has two blocks of information and the right panel has four blocks. The configuration of such block states has vanishing average (global) overlap with the original pattern. However, the structured distribution of local overlaps carries some spatially ordered information, the blocks oscillating between negative and positive overlaps. In other words, it is possible to recognize the image even if the information about the original pattern is zero. We call this a local retrieval, to distinguish it from the usual global retrieval of the full pattern.

The existence of locally organized memories rises some questions that we have attempted to answer. First, can stable block states emerge spontaneously in an associative network? Second, how can be measured the information hidden in these blocks? Third, which neural architectures are able to convert this local information into global information?

Unlike previous studies about structured information in memory networks (bumps) [14,15], we have considered the simplest model of binary uniform neurons, with small-world connectivity and without any reinforcement mechanism. In this way we can single out the effect of topology on the structure of the retrieval attractor. In addition to this issue, we have considered nontrivial block structures. We will show that blocks of memories can appear spontaneously as a consequence of neural dynamics combined with the network topology. We have also studied the conditions, for the topology of synaptic connectivity, in which local overlaps are either stable or unstable and in which cases they help to retrieve

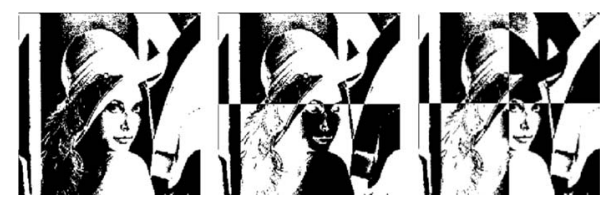

FIG. 1. Image with different spatial distributions of overlaps. Left: Original picture. Center: two-blocks. Right: four-blocks. Both spatial distributions, two-blocks and four-blocks, have null global overlap with the original picture. 
complete patterns. In addition, we propose a method to measure local information and compare it with the global information.

The emergence of spatial structures is of interest in computational neuroscience, for example, when considering that distinct sensory organs receiving independent stimuli manage to generate a unified response [16]. In particular, the role of the columnar structure of the neocortex has been the subject of much recent research, see Ref. [17], and references therein. Indeed, the cortical model with biologically imposed constraints behaves as a modular attractor memory, which improves its representational abilities while complying with experimental data.

In the next section we describe the topology of the network and its neural dynamics, as well as proposing a measure for the local information. In the third section we identify the topological conditions for the transition between phases of local and global retrieval, both by simulation and theory.

\section{THE MODEL}

\section{A. Topology and dynamics}

At any given time $t$, the network state is defined by a set of binary neurons $\vec{\sigma}^{t}=\left\{\sigma_{i}^{t} \in \pm 1, i=1, \ldots, N\right\}$. The purpose of the network is to recover a set of independent patterns $\left\{\vec{\xi}^{\mu}, \mu=1, \ldots, P\right\}$ that have been stored by a learning process. Each pattern, $\vec{\xi}^{\mu}=\left\{\xi_{i}^{\mu} \in \pm 1, i=1, \ldots, N\right\}$, is a set of siteindependent unbiased binary random variables $p\left(\xi_{i}^{\mu}= \pm 1\right)$ $=1 / 2$.

The synaptic couplings between the neurons $i$ and $j$ are given by the adjacency matrix $J_{i j} \equiv C_{i j} W_{i j}$, where the topology matrix $\mathbf{C}=\left\{C_{i j}\right\}$ describes the connection structure of the neural network and in $\mathbf{W}=\left\{W_{i j}\right\}$ are the learning weights. The topology matrix is split into local and random links. The local links connect each neuron to its $K_{l}$ nearest neighbors in a closed ring, while the random links connect each neuron to $K_{r}$ others uniformly distributed in the network. Hence, the network degree is $K=K_{l}+K_{r}$. The network topology is then characterized by two parameters, the connectivity ratio and the randomness ratio, respectively, defined by

$$
\gamma=K / N, \quad \omega=K_{r} / K,
$$

where $\omega$ plays the role of a rewiring probability in the smallworld model [6]. An extremely diluted network is obtained as $\gamma \rightarrow 0$, and the storage cost of this network is $|\mathbf{J}|=N \times K$ if the matrix $\mathbf{J}$ is implemented as an adjacency list of $K$ neighbors.

The task of the network is to retrieve a pattern (say, $\vec{\xi}$ $\left.\equiv \vec{\xi}^{\mu}\right)$ starting from a neuron state $\vec{\sigma}^{0}$ which is close to it. This is achieved through the neuron dynamics

$$
\begin{gathered}
\sigma_{i}^{t+1}=\operatorname{sgn}\left(h_{i}^{t}\right), \\
h_{i}^{t} \equiv \frac{1}{K} \sum_{j} J_{i j} \sigma_{j}^{t}, \quad i=1, \ldots, N,
\end{gathered}
$$

where $h_{i}^{t}$ denotes the local field of neuron $i$ at time $t$. We used stochastic asynchronous updating in the present work, except in Sec. III D and Fig. 6, where parallel dynamics were used to compare simulations with theory. Stochastic macrodynamics take place due to the extensive learning of $P=\alpha K$ patterns, where $\alpha$ is the load ratio. The weight matrix $\mathbf{W}$ is updated according to the Hebb's rule

$$
W_{i j}^{\mu}=W_{i j}^{\mu-1}+\xi_{i}^{\mu} \xi_{j}^{\mu} .
$$

Weights start at $W_{i j}^{0}=0$ and after $P$ learning steps, they reach the value $W_{i j}=\Sigma_{\mu}^{P} \xi_{i}^{\mu} \xi_{j}^{\mu}$. The learning stage displays slow dynamics, being stationary within the time scale of the faster retrieval stage Eq. (2).

\section{B. The information measures}

Previous studies have only dealt with global measures of information, which are adequate to describe networks with no local connectivity. For small-world connectivity it is useful to define blocks as the structured pieces of information that emerge in the network. If the contiguous neurons are distributed within $b$ blocks, for simplicity each of size $L$ $=N / b$, then the block's overlap between the neural states and one individual pattern restricted to the $l$ th block ( $l$ $=1, \ldots, b)$ is

$$
m_{l} \equiv \frac{1}{L} \sum_{i \in l} \xi_{i} \sigma_{i},
$$

at an unspecified time step. We can consider $m_{l}$ as a random variable and estimate the average of this variable across the blocks as $\left\langle f_{l}\right\rangle_{b} \equiv \frac{1}{b} \sum_{l=1}^{b} f_{l}$.

The relevant order parameters measuring quality of retrieval are the mean $(m)$ and the variance $(v)$ of the block overlap distribution, defined as

$$
m \equiv\left\langle m_{l}\right\rangle_{b}, \quad v \equiv\left\langle m_{l}^{2}\right\rangle_{b}-m^{2} .
$$

Note that $m$ is the usual global overlap, also written as $m$ $\equiv \frac{1}{N} \sum_{i} \xi_{i} \sigma_{i}$. When the global overlap is zero and the size of the blocks is taken as $L=1$, the network carries no macroscopic order. On the other hand, if there is only one block, $b=1$, the variance is zero and no local information is carried. However, if the size is large but $1 \ll L<N$, the variance is finite and the blocks convey only local information. The standard deviation, which we name the local overlap, is $\delta$ $=\sqrt{v}$. It is worth mentioning that the blocks are macroscopically scaled and hence the parameter $\delta$ is not related to a spin glass, which is a microscopic ordering.

Together with the overlap, the load ratio $\alpha \equiv P / K$, that accounts for the storage capacity, is needed. As the number of stored patterns grows the network is not able to retrieve them and the overlap goes to zero. To fully describe the performance of a structured network with a unique measure, it is useful to use tools from information theory [4]. Let us first calculate the global mutual information $\mathcal{M}$, a quantity used to measure the information that an observer can receive at the output of a channel. The recall process of stored patterns that we are considering here can be regarded as a channel, with the pattern as the input and the neuron states as the output. The mutual information can be defined in terms of the order parameter $m=\langle\sigma \xi\rangle_{\sigma, \xi}$, in the limits $K, N \rightarrow \infty$. The 

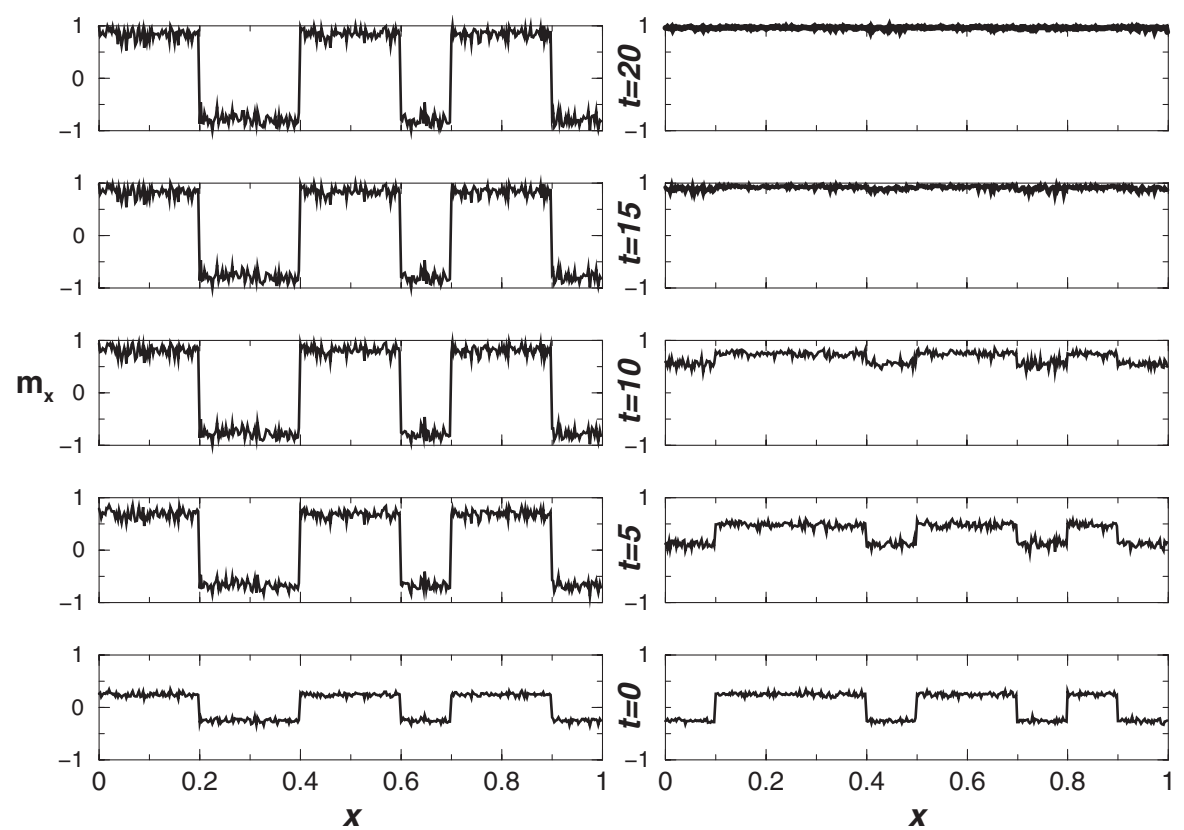

FIG. 2. Evolution of blocks with $m_{l}^{0}= \pm 0.3$, from $t=0$ (bottom) to $t=20$ (top), for $\gamma=10^{-4}$, with $N=10^{6}$. Left: $\omega=0.1, \alpha$ $=0.05$. Right: $\omega=0.5, \alpha=0.20, x$ $\equiv i / N$. brackets represent an average over the joint distribution $p(\sigma, \xi)$ for a single neuron, which is understood as an ensemble distribution for the neuron states $\left\{\sigma_{i}\right\}$ and patterns $\left\{\xi_{i}^{\mu}\right\}$. This will give $\mathcal{M}[\sigma ; \xi]=S[\sigma]-S[\sigma \mid \xi]$, with the entropy of the output channel $S[\sigma]=1[$ bit $]$ and the conditional entropy obeying [9]

$$
S[\sigma \mid \xi]=-\frac{1+m}{2} \log _{2} \frac{1+m}{2}-\frac{1-m}{2} \log _{2} \frac{1-m}{2} .
$$

Hence we define the global information ratio as

$$
i_{m}(\alpha, m) \equiv \alpha \mathcal{M}[\sigma ; \xi],
$$

for independent neurons and uncorrelated patterns. The global information ratio is useful to evaluate the network performance when there is a global stimulus [4]. Below, we estimate the local information due the distribution of blocks when the global overlap is $m=0$.

We consider a sample of $b$ independent blocks of pattern overlaps, their distribution being described by their mean $m$ and variance $v$. The local information can be estimated from a Gaussian channel with the output state comprised of a signal term $m_{l}$ with variance $v$ and a noise term $z$, whose variance is assumed to be $v_{z} \sim 1$ (maximal for the signal). Thus, the mutual information satisfies $\mathcal{M}\left[\vec{\sigma},\left\{\vec{\xi}^{\mu}\right\}\right] \sim S\left[m_{l}+z\right]$ $-S[z]$ and the local information ratio is roughly [18]

$$
i_{v}\left(\alpha, m_{l}\right) \simeq \alpha \log _{2}(1+v) .
$$

It should be noted that the underlying block distribution is unknown, except its first and second moments. The approximation in Eq. (9) supposes that the block distribution is Gaussian, and therefore it provides an upper bound estimation of the local information. The estimation for $i_{v}$ is not as exact as the expression for the global information $i_{m}$. Nevertheless, it works well when $v=0$ (all blocks have same overlap), such that $i_{v}=0$ and there is only global information $i_{m}$ $\geqslant 0$. Moreover, it also scales well with $v$ in the case of per- fect blocks $(v=1)$, since the information regarding the blocks corresponds to $i_{v}=\alpha$ bits. Note that if no spatial correlation emerges, $m_{l}$ provides no information at all and it can be regarded as pure noise (both $i_{m}=0$ and $i_{v}=0$ ). Equation (9) holds as long as the block is sufficiently large, $L \gg 1$. Both global and local information are not manipulated jointly in a unified formula, and therefore, they are analyzed separately.

The validity range for each expression in Eqs. (8) and (9), for the global and local information, are either the global $(\delta=0)$ or the local retrieval regime $(m=0)$, respectively. Finally, note that the global and local overlaps evolve with time according to the neuron dynamics Eq. (2), such that at each step they have a time index $m^{t}$ and $\delta^{t}$.

\section{RESULTS}

\section{A. Simulations: the retrieval evolution}

We simulated the dynamical neuron equations (2)-(4) with the topology parameters defined according to Eq. (1). The block overlaps time evolution is illustrated in Fig. 2 for a network of $N=10^{6}$ neurons distributed in $b=10$ blocks and with connectivity $\gamma=10^{-4}$. The initial block overlaps were chosen at random from a discrete uniform distribution $m_{l}^{t=0}$ $= \pm 0.3$. In the left panel $\alpha=0.05$ and $\omega=0.1$, in the right panel $\alpha=0.20$ and $\omega=0.5$. To improve the clarity of the figures, the neuron overlaps $m_{i}$ have been smooth averaged across uniform windows (of size $\delta L=10^{3}$ ) inside the blocks (of size $L=10^{5}$ ). Hence the plotted curves $m_{x}$, with $x=i / N$ are smoother than the actual $m_{i}$, although some of the structure can still be appreciated in $m_{l}$. In these plots time evolves from $t=0$ (bottom) to $t=20$ (top), which is close to the stationary state. The left panels show that the network retains its initial block configuration: the blocks are retrieved as independent patterns increasing their overlaps to the fixed point $m_{l}^{*} \sim \pm 1.0$. In the right panel, the blocks lose their starting signals and the full pattern is completed, $m^{*} \sim 1.0$. While for local topology $(\omega=0.1)$ the block structure persists, for ran- 


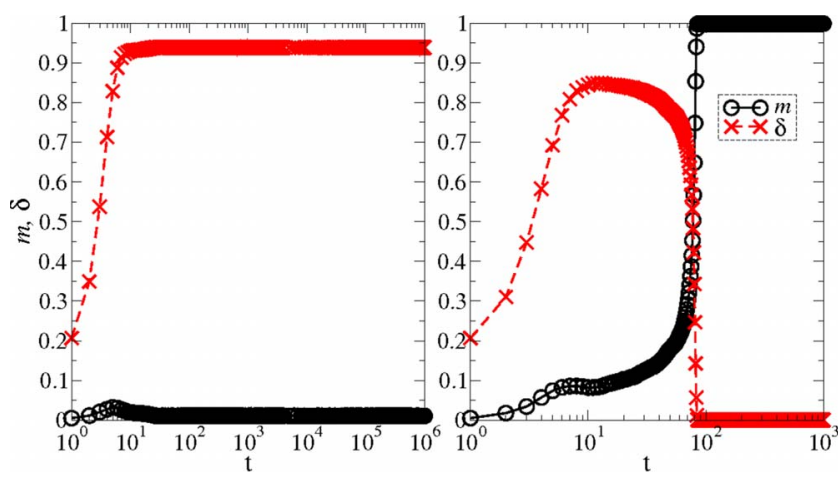

FIG. 3. (Color online) Network evolution, with $N=10^{6}, \gamma$ $=10^{-4}, \omega=0.3$. Left: $B$ phase, $\alpha=0.1$. Right: $R$ phase, $\alpha=0.2$.

dom topology $(\omega=0.5)$ the local information is converted into global information.

The time evolution of a network starting in blocks with $m_{l}^{t=0} \simeq \pm 0.2$ is depicted in Fig. 3. The randomness is $\omega$ $=0.3$. For a low load ratio $\alpha=0.1$, the network maintains its block configuration and the local overlap fluctuates slightly around the fixed point $\delta^{*} \sim 0.94$, as seen in the left panel. For a larger load $\alpha=0.2$, the network evolves to the global attractor in a more complex way, as seen in Fig. 3 right. Initially the network approaches the local state, improving the blocks' overlap $\left(m^{t} \sim 0, \delta^{t} \sim 0.8\right)$. After $t=90$ time steps, the network quickly loses its block structure, and evolves into a global state with the completion of the learned pattern $m \approx 1$. We have checked for the stability in both cases, by running the simulation for up to $t=10^{6}$ time steps. Although there may be microscopic cycles, the macroscopic state converges to either the local or the global retrieval phase [19].

\section{B. Simulations: Learning capacity}

We studied the stationary states of the network as a function of the load ratio $\alpha$ for different values of the topological parameter $\omega$. A sample of the simulation results is shown in Fig. 4. The stationary global and local overlaps $m^{*}$ and $\delta^{*}$, respectively, are plotted in the top panels for $\gamma=10^{-3}$ and randomness ranging from $\omega=0.0$ to $\omega=1.0$. The network starts in a block configuration with $b=10, m^{0}=0$, and $\delta^{0}=1$. We observed that the global overlap remains close to $m^{*}$ $\sim 1$ for random networks, while for local networks the local overlap increases to $\delta^{*} \sim 1$, up to the respective global and local critical capacities.

One reason for this behavior is that randomness decreases the mean-path length between neurons, facilitating the propagation of the information around the network and yielding a global ordering. On the other hand, locality increases the clustering of neurons, slowing down the transmission of information across the network and stabilizing the

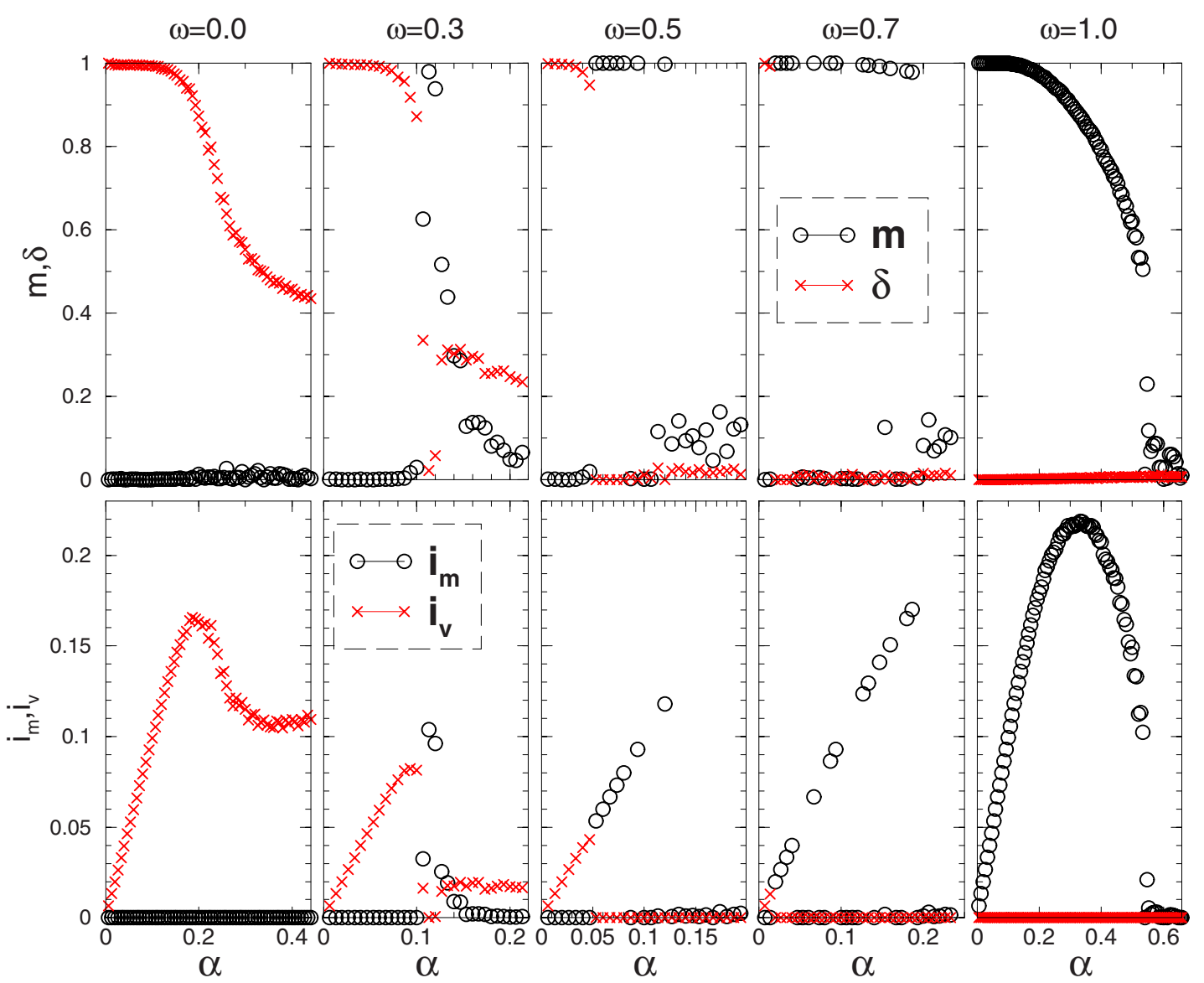

FIG. 4. (Color online) Global and local overlaps $m, \delta$ (top) and information $i_{m}, i_{v}$ (bottom), vs $\alpha$, for $b=10, \gamma=10^{-3}$, and $\omega$ from 0.0 (left) to 1.0 (right). Networks with $N=3 \times 10^{5}$ and initial states $m^{0}=0, \delta^{0}=1$. 
formation of the blocks. It is worth noting that the network can retrieve the full pattern achieving $m^{*} \sim 1$ even starting at $m^{0}=0$, thanks to the role of the topology and the \pm block overlaps,.

There is also a critical load for the global and local overlaps $\alpha_{R}$ and $\alpha_{B}$, respectively. For instance, in the middle panel $(\omega=0.5)$ the local overlap decays sharply to $\delta^{*}=0$ at $\alpha_{B} \sim 0.05$, coinciding with an increase of the global overlap to $m^{*} \sim 1$. Later in the learning process (increasing further on the load ratio), at $\alpha_{R} \sim 0.11$, it establishes a second transition for the global overlap close to $m=0$.

Figure 4 (bottom) displays the global and the local information as function of the load ratio. The panels show that both local and global are a nonmonotonic function of the load ratio. It can be seen that the maximal local (global) information $i_{v}\left(i_{m}\right)$ decreases (increases) with the values of $\omega$. Indeed, the maximum of the local information $i_{v} \sim 0.17$ for a local topology $\omega=0.0$ is comparable to the maximum of the global information $i_{m} \sim 0.22$ for a random topology $\omega$ $=1.0$. Intermediate randomness (middle panels) led to competition between blocks and global structures. Indeed, above some load critical ratio $\alpha_{B}$, the blocks lose stability and the neurons shift to the global information mode.

\section{Phase Diagram}

According to the definitions of the order parameters, and following the results presented in the previous sections, we define the stationary states of this small-world ANN. The network may exhibit either a global retrieval $(R)$ phase, with $m \neq 0, \delta=0$, or a local retrieval $(B)$ phase, with $m=0, \delta>0$, which carries nonvanishing information. Furthermore, there is a zero $(Z)$ phase, with $m=0$ and $\delta=0$, without any information. When the network starts in the vicinity of a pattern, it will move closer to that pattern if the load ratio is lower than that of the global retrieval saturation $\alpha_{R}(\omega)$. When the blocks of a network start successively near to a pattern or the inverse of that pattern, the block configuration will persist if the load is lower than the local retrieval saturation $\alpha_{B}(\omega)$. For large $\omega$, the stable phase is the $R$ whereas for small $\omega, B$ is the stable phase.

In order to study these phases, extensive Monte Carlo simulations of the system were performed, with $K=100$ and $N=10^{6}$ neurons $\left(\gamma=10^{-4}\right)$. The phase diagram is shown in Fig. 5, for a network with $b=2$ blocks. The initial condition for the $B$ phase is $m^{0}=0$ and $\delta^{0}=1$. The separation between the $R_{1}$ and $R_{0}$ phases (the thin dashed line) is justified by their distinct dynamical character: while $R_{1}$ is the usual retrieval attractor for initial conditions $m^{0} \neq 0, \delta^{0}=0$, the $R_{0}$ is the retrieval attractor when the network starts with no initial global overlap $m^{0}=0, \delta^{0} \neq 0$.

There is a transition from the $Z$ to $R$ phases at a $\alpha_{R}(\omega)$, represented by the thick dashed line, below which the global retrieval information is always stable. The local information appears at the line $\alpha_{B}(\omega)$ (solid line), below which the $B$ phase coexists with $R$. We could see that the $B$ region holds steady at a larger $\alpha$ for local networks than for random networks. We also noted that mixed states, with both $m \neq 0, \delta$ $\neq 0$, also emerged from no pure $R$ or $B$-like initial condi-

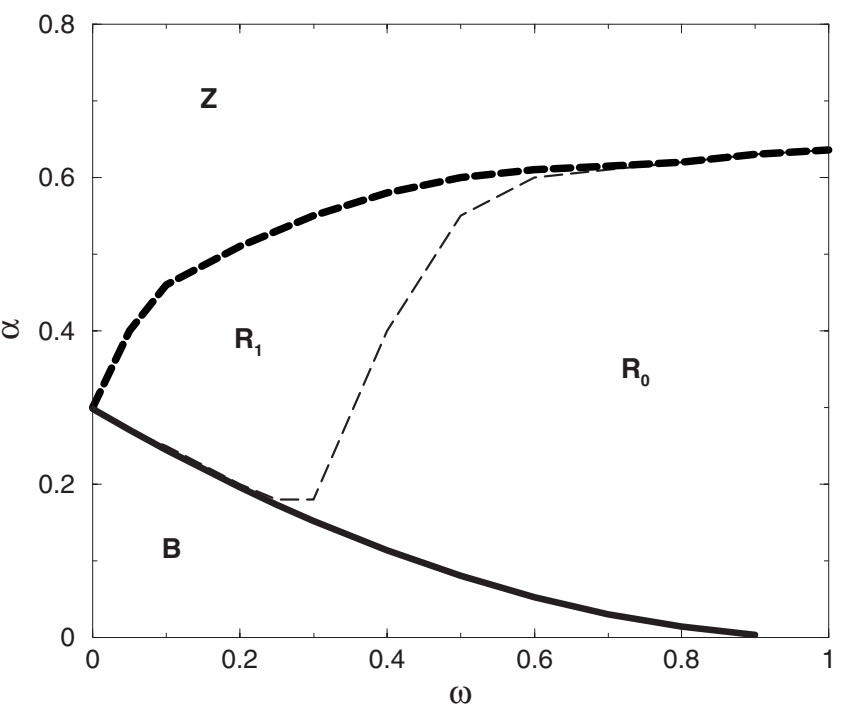

FIG. 5. Phase diagram $(\omega \times \alpha)$ for $N=10^{6}, \gamma=10^{-4}$, and $b=2$. $B \equiv$ Local phase. $R_{0}\left(R_{1}\right) \equiv$ retrieval phase with $m^{0}=0, \delta^{0}=1 \quad\left(m^{0}\right.$ $\left.=1, \delta^{0}=0\right)$. $Z \equiv$ no information.

tions, as seen in Fig. 2. We checked through extensive simulations that the local solution is robust for a wide range of numbers of blocks, and we found that the transition $\alpha_{B}(\omega)$ in the phase diagram of Fig. 5 shrinks to zero for $b>10^{3}$. The $R$ region also collapses to the $\alpha=0$ axis and only the $Z$ phase survives.

\section{Theory}

In this section we consider a strongly diluted network and we propose theoretical equations for the macroscopic order parameters. The sketch of a proof that is valid for stationary equations can be found in the Appendix. Here we add empirical dynamical equations whose fixed points coincide with the stationary solutions. This extrapolation is justified for parallel dynamics, for which all neurons are updated once at each time step. We suppose that the neurons are distributed within $b$ blocks, with positive and negative overlaps, $m_{l}$ $\equiv m_{ \pm}$. Hence the global overlap is $m=\left(m_{+}+m_{-}\right) / 2$ and the fluctuation between blocks is $\delta=\left(m_{+}-m_{-}\right) / 2$.

An approximation for the local field of neurons at block $m_{l}$ at time step $t$ gives

$$
\xi h_{l}^{t} \equiv \omega m^{t}+(1-\omega)\left(m^{t}+y_{l} \delta^{t}\right)(1-\gamma b)+\Omega^{t},
$$

where $\xi$ is the pattern being retrieved. The patterninterference noise follows a Gaussian distribution $\Omega$ $\doteq N(0, \alpha r)$, where $r=\omega r_{r}+(1-\omega) r_{l}$ is the sum of the random and local feedback terms $r=\omega r_{r}+(1-\omega) r_{l}$, with $r_{r}=1$ and $r_{l}=(1-\chi)^{-2}$. The susceptibility $\chi$ arises from the local connections

$$
\chi^{t}=\frac{1}{\sqrt{\alpha r_{l}}}\left\langle z \operatorname{sgn}\left(\xi h^{t}\right)\right\rangle_{y, z} .
$$

With the field in Eq. (10), the macrodynamics for the global and local overlap are 


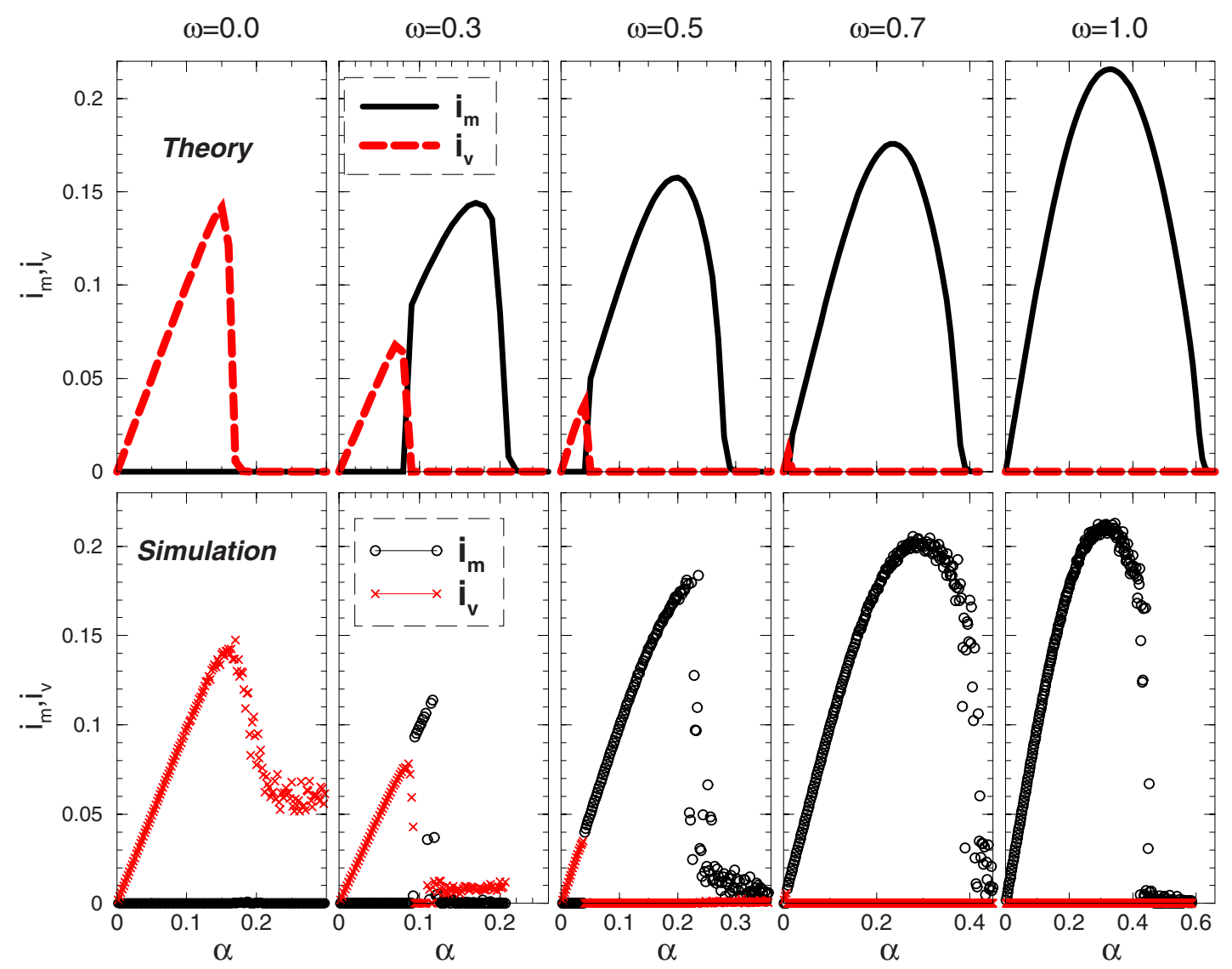

FIG. 6. (Color online) Global (solid lines and circles, $i_{m}$ ) and local (dashed lines and crosses, $i_{v}$ ) information, vs $\alpha$, for $b=2$. Randomness runs from $\omega$ from 0 (left) to 1 (right), $\gamma=10^{-2}$. Top: theory. Bottom: simulation with $N=10^{5}, K=10^{3}$, and parallel dynamics. Initial states: $m^{0}=0.04 ; \delta^{0}=1$.

$$
\begin{aligned}
& m^{t+1}=\left\langle\operatorname{sgn}\left(\xi h^{t}\right)\right\rangle_{y, z}, \\
& \delta^{t+1}=\left\langle y \operatorname{sgn}\left(\xi h^{t}\right)\right\rangle_{y, z} .
\end{aligned}
$$

The averages are over the binary distribution of $y \in \pm 1$ and a Gaussian $z \doteq N(0,1)$. There are two types of stationary states: (1) $m \neq 0, \delta=0$, with $m=\operatorname{erf}\left(m / \sqrt{2 \alpha r_{l}}\right)$ and (2) $m=0$, $\delta>0$, with $\delta=\operatorname{erf}\left[\delta(1-\omega) / \sqrt{2 \alpha r_{l}}\right]$. The first is the usual Amit's solution [1], while the second is the local solution, which is stable if $(1-\omega) \geqslant \sqrt{\pi \alpha r / 2}$. Adjusting the solid curve $\alpha_{B}$ in the Fig. 5 to $(1-\omega) \approx A_{0} \alpha^{A_{1}}$, gives $A_{1}=0.51$, which fits well with this theoretical prediction if one assumes $r$ is constant in the transition.

A comparison between the theoretical results (upper panels) and the simulation (bottom panels) for parallel dynamics is shown in Fig. 6. Qualitatively, the behaviors predicted by theory, of local retrieval with small $\omega$ and global retrieval with large $\omega$, agree quite well with that checked by simulations. The transition from local to global retrieval at a given $\alpha(\omega)$ (for intermediate randomness $0.3 \leqslant \omega \leqslant 0.7$ ), as well as the maximal local and global information, are also in agreement. Moreover, Eq. (10) explains why local retrieval fails for $L \sim K$, for which $\gamma b \sim 1$ and the boundary effects between blocks are relevant when compared to the bulk of the connected neurons. The degree of dilution plays the role of a resolution scale for the boundary effects. Therefore, only diluted networks are able to stabilize blocks.

Finally note that both the theoretical results and the simulations start with $m^{0}=0.04$, because $m^{*} \neq 0$ would never be achieved if the initial global overlap is zero, as we observed in the simulation for asynchronous update (see Fig. 4). The theoretical equations for the asynchronous dynamics are more involved than the parallel macrodynamics and they lead to differential equations, the calculation of which is beyond the scope of this work.

Except for this different dynamical behavior, both parallel and asynchronous updating produce similar attractors. It is worth comparing the simulation results of the lower panels of Fig. 6 with those of Fig. 4. First, it should be noted that they were calculated for different values of the network parameters (see the figure legends). For instance, in Fig. 6 the connectivity is $K=10^{3}$ while in Fig. 4 it is $K=300$. The pattern storage capacity is related to $K$, hence the Fig. 6 displays more points for each learned pattern. In Fig. 4 the networks are also more diluted $\left(\gamma=10^{-3}\right)$ than in Fig. $6\left(\gamma=10^{-2}\right)$, which should lead to a larger storage capacity for the global overlap in Fig. 4 than in Fig. 6 [4]. However, with a $\omega$ $=0.5$ and $\omega=0.7$ in both panels, the more diluted network (Fig. 4) reaches a smaller critical load $\alpha_{R}$. In fact, there are many holes in the curve that represent failures in the recovery of the pattern. The reason for this disagreement with the expected result is that the network has only $b=2$ blocks in 
Fig. 6, in contrast with the $b=10$ blocks in Fig. 4. The more blocks that the network is initialized with, the more difficult it is to complete the pattern. This occurs because the borders between each block make them less stable.

\section{CONCLUSIONS AND DISCUSSION}

In this paper we have studied a type of solution for an attractor neural network: the local retrieval phase $(B)$ with overlaps structured in blocks. The dependence of the stability of the local $B$ and the global retrieval $(R)$ phases on the topological parameters of connectivity $(\gamma)$ and randomness $(\omega)$ was analyzed. Although the storage capacity is severely disrupted when the long-range nature of the connections is lost, we found that local information emerges when the network has a more local topology. The local information corresponds to configuration states which carry information in blocks of neighboring neurons, and are attractors of the network dynamics. A block structure might resemble the metastable mixed states studied in the seminal work of Amit [1]. These spurious states, where the network only recognizes mixture of patterns, may provide useful information, as might the $B$ phase, because the blocks are spatially ordered. However, while the local field arising from different patterns can cooperate to retrieve both of them, the local field induced by negative and positive patterns cancels each of these out, and the blocks are unstable in networks without the type of topology studied here.

Both in biology and in hardware implementations of neural systems, mainly neighboring neurons are connected in networks. Such short-range architectures are much cheaper in terms of the wiring cost than long-range ones, but the downside is that the network loses most of its global retrieval capacity. In this paper we have shown that such a structure induces another information retrieval capacity: that of local retrieval. Another novel situation that arises from this memory structure is that the information from blocks may be transferred to a global retrieval if the range of links is long enough, or if more patterns are stored. Hence, the topology complexity improves the retrieval attractor basin. We found that the transition from $R \rightarrow B$ takes place for $\alpha \leqslant \alpha_{B} \approx(1$ $-\omega)^{2}$, and we propose a theory for strongly diluted networks, which fits well with our simulations. We also estimated the information entropies, for both $R$ and $B$ phases.

The blocks behave as independent pieces of information. Thus, instead of the small number $P$ of patterns of size $N$ that a diluted network can store, this phase is able to retrieve $b$ $\times P$ patterns, each with size $N / b$. We believe that the existence of an information phase with no global overlap may play a relevant role in natural neural networks, for instance, to manage a successful response to stimuli activating separated cortical areas [20]. Also in many applications of pattern classification, such as image recognition, by carrying local spatial information the overlaps may have opposite signals in separate blocks, although overall information might be generated. Minor changes in the topology $\mathbf{C}$, for instance, that suppress symmetry constraints, will lead to complex dynamics for the blocks, including cycles and chaos, which could model higher functions of the brain [21]. A more detailed study of the block distribution also warrants further investigation.

\section{ACKNOWLEDGMENTS}

This work was supported by the MEC Grants No. TIN2004-04363-CO03-03, No. TIN-2007-65989 and by the CAM Grant No. S-SEM-0255-2006. E.S. was partially supported by the MEC Grant No. PR2007-0080. We thank K. Koroutchev and R. Levi for useful discussion.

\section{APPENDIX: MACRODYNAMIC EQUATIONS}

Let the neurons be randomly distributed within $b$ blocks and for simplicity, each of size $L=N / b$, with positive and negative overlaps $m_{l}=m_{ \pm}$. The blocks $l=1, \ldots, b$ are built as the sets $\lambda_{l}=\{i=(l-1) L+k ; k=1, \ldots, L\}$. Then, according to Eq. (6) the global overlap is $m=\sum_{l}^{b} m_{l} / b$ and the fluctuation between blocks is $\delta=\sqrt{v}$, with $v \equiv \sum_{l}^{b} m_{l}^{2} / b-m^{2}$. The block's overlap can be written as

$$
m_{l}=m+y_{l} \delta,
$$

where $y_{l} \doteq \pm 1$ (according to the block) is a random variable.

The local field of the neuron $\sigma_{i}$, Eq. (3), applying Eq. (4) for the weights, can be separated into a signal and a noise term, if a given pattern is being retrieved, say $\xi \equiv \xi^{\mu=1}$ :

$$
h_{i} \equiv \xi_{i} m_{i}+\Omega_{i}
$$

where

$$
m_{i}^{\mu} \equiv \frac{1}{K} \sum_{j \in C} \xi_{j}^{\mu} \sigma_{j}, \quad \Omega_{i} \equiv \sum_{\mu>1} \xi_{i}^{\mu} m_{i}^{\mu}
$$

are the overlap restricted to the neighbors $C$ of neuron $\sigma_{i}$, and the cross-talk noise, respectively.

There are local and random neighbors for each neuron, hence the signal term itself splits into localized and randomized terms, namely,

$$
m_{i}=\frac{K_{r}}{K} m_{i}^{r}+\frac{K_{l}}{K} m_{i}^{l},
$$

with $m_{i}^{x} \equiv \frac{1}{K_{x}} \sum_{j \in C_{x}} \xi_{j} \sigma_{j},(x \equiv l, r)$ where $K_{l}^{i}$ and $K_{r}^{i}$ are the local and random sets of neighbors of the neuron $\sigma_{i}$, respectively.

From Eq. (5), whenever the neighbors belong to a block the local field depends on its block's overlap $m_{l}$. On the other hand, the randomized field is a sample of a global field that does not depend on a block. Using the definition in Eq. (1), one arrives at an approximation for the local field of neurons in the block $\lambda_{l}$

$$
\xi h_{l} \equiv \omega m+(1-\omega)\left(m+y_{l} \delta\right)(1-\gamma b)+\Omega,
$$

where $\xi$ is the pattern being retrieved. The correction term $(1-\gamma b)$ accounts for the boundary effects between $m_{ \pm}$ blocks.

The equation for the block's overlap is then $m_{l}$ $=\langle\operatorname{sgn}(\xi h)\rangle_{\Omega}$, where the average in the angular brackets are 
over the noise $\Omega$. But from Eq. (A1) $m_{l}=m+y_{l} \delta$, and thus, after averaging over $y_{l}$ one gets

$$
\begin{gathered}
m=\left\langle m_{l}\right\rangle_{y}=\langle\operatorname{sgn}(\xi h)\rangle_{y, z}, \\
\delta=\left\langle y m_{l}\right\rangle_{y}=\langle y \operatorname{sgn}(\xi h)\rangle_{y, z} .
\end{gathered}
$$

The average over $z$ stands for the noise distribution $\Omega$.

This noise is a large sum of almost-independent terms, which converges to a Gaussian distribution $\Omega \doteq N(0, \Delta)$ [7]. Its variance $\Delta=\alpha r$ is given by the sum of random and local feedback terms $r=\operatorname{var}\left(m_{i}^{\mu}\right)=\omega r_{r}+(1-\omega) r_{l}$. To deal with them, one can consider the residual overlaps $(\mu>1)$ as stochastic variables. If one expands the residual overlaps around $h_{j}^{\mu} \equiv h_{j}-\xi_{i}^{\mu} m_{i}^{\mu}$ it holds that

$$
m_{i}^{\mu} \sim \frac{1}{K} \sum_{j \in C} \xi_{j} \operatorname{sgn}\left(h_{j}^{\mu}\right)+m_{i}^{\mu} \chi_{i},
$$

$$
\chi_{i} \equiv \frac{1}{K} \sum_{j \in C} \frac{d}{d h_{j}^{\mu}} \operatorname{sgn}\left(h_{j}^{\mu}\right),
$$

where $\chi_{i}$ is the susceptibility. The first term on the right-hand side of Eq. (A7) is not correlated with the second term, and its variance is $\alpha$. So, the stochastic equation reads $m_{i}^{\mu}(1$ $-\chi) \simeq N(0, \alpha)$, and the feedback term is

$$
r_{x}=\left(1-\chi_{x}\right)^{-2}, \quad x=l, r .
$$

Let us now suppose only strongly diluted networks $(K$ $\ll N)$. For random connections, $\chi_{r}$ can be neglected since there is no feedback in the dynamics and $r_{r}=1$ [9]. However, for local connections, even extreme dilution does not eliminate the feedback and thus, susceptibility can also be written as

$$
\chi_{l}=\frac{1}{\sqrt{\alpha r_{l}}}\langle z \operatorname{sgn}(\xi h)\rangle_{y, z} .
$$

[1] D. J. Amit, Modelling Brain Function: The World of Attractor Neural Networks (Cambridge University Press, Cambridge, 1989).

[2] C. Li and G. Chen, Phys. Rev. E 68, 052901 (2003).

[3] P. N. McGraw and M. Menzinger, Phys. Rev. E 68, 047102 (2003).

[4] D. Dominguez, K. Koroutchev, E. Serrano, and F. B. Rodriguez, Neural Comput. 19, 956 (2007).

[5] R. Albert and A. Barabasi, Rev. Mod. Phys. 74, 47 (2002).

[6] D. J. Watts and S. H. Strogatz, Nature (London) 393, 440 (1998).

[7] J. Hertz, J. Krogh, and R. Palmer, Introduction to the Theory of Neural Computation (Addison-Wesley, Boston, 1991).

[8] M. Okada, Neural Networks 9, 1429 (1996).

[9] D. R. C. Dominguez and D. Bolle, Phys. Rev. Lett. 80, 2961 (1998).

[10] N. Masuda and K. Aihara, Biol. Cybern. 90, 302 (2004).

[11] T. Nikoletopoulos, A. C. Coolen, I. P. Castillo, N. S. Skantzos, J. P. Hatchett, and B. Wemmenhove, J. Phys. A 37, 6455 (2004).
[12] J. P. L. Hatchett, I. Perez Castillo, A. C. C. Coolen, and N. S. Skantzos, Phys. Rev. Lett. 95, 117204 (2005).

[13] L. Morelli, G. Abramson, and M. Kuperman, Eur. Phys. J. B 38, 495 (2004).

[14] Y. Roudi and A. Treves, Phys. Rev. E 73, 061904 (2006).

[15] K. Koroutchev and E. Korutcheva, Phys. Rev. E 73, 026107 (2006).

[16] E. Rolls and A. Treves, Neural Networks and Brain Function (Oxford University Press, Oxford, 1998).

[17] C. Johansson and A. Lansner, Neural Comput. 19, 1871 (2007).

[18] T. M. Cover and J. A. Thomas, Elements of Information Theory (John Wiley, New York, 1991).

[19] D. R. Paula, A. D. Araujo, J. S. Andrade, H. J. Herrmann, and J. A. C. Gallas, Phys. Rev. E 74, 017102 (2006).

[20] Y. Roudi and A. Treves, J. Stat. Mech.: Theory Exp. (2004) P07010.

[21] A. Damasio, Descartes' Error: Emotion, Reason, and the Human Brain (Grosset/Putnam, New York, 1994). 